\title{
Comparação da assistência em saúde mental em unidades básicas de saúde com ou sem equipe do Programa de Saúde da Família
}

\author{
Comparison of mental health assistance in primary care settings with or without Family \\ Health Program team
}

\author{
Mário Sérgio Ribeiro ${ }^{1}$, Márcio José Martins Alves ${ }^{2}$, Priscila Matthiesen e Silva ${ }^{3}$, \\ Eveline Maria de Melo Vieira ${ }^{4}$
}

\begin{abstract}
${ }^{1}$ Doutor em Filosofia. Médico, Professor associado de Psiquiatria, Faculdade de Medicina, Universidade Federal de Juiz de Fora (UFJF), Juiz de Fora, MG. ${ }^{2}$ Doutor em Saúde Coletiva. Médico. Professor adjunto de Saúde Coletiva, Faculdade de Medicina, UFJF. ${ }^{3}$ Acadêmica de Medicina. Auxiliar de pesquisa, bolsista Fundação de Amparo à Pesquisa do Estado de Minas Gerais (FAPEMIG). ${ }^{4}$ Acadêmica de Medicina, auxiliar de pesquisa.

Este estudo foi realizado no Laboratório de Pesquisas em Personalidade, Álcool e Drogas (LAPPDA), Faculdade de Medicina, Universidade Federal de Juiz de Fora (UFJF), Juiz de Fora, MG.
\end{abstract}

\section{Resumo}

Introdução: O objetivo deste estudo foi comparar o perfil de assistência em saúde mental realizado por unidade básica de saúde (UBS) com equipe de Programa de Saúde da Família (PSF) e sem equipe de PSF.

Método: Estudo observacional, avaliando pacientes encaminhados por UBS da área de abrangência de um serviço especializado de saúde mental no período de abril de 2003 a março de 2006.

Resultados: A UBS com equipe de PSF apresentou melhor padrão global de registros de dados, maior responsabilidade exclusiva do médico em suas referências ao nível especializado $(\mathrm{p}=0,000)$, menor capacidade de retenção dos usuários na UBS $(\mathrm{p}=0,099)$, maiores taxas de abandono de tratamento em nível secundário $(\mathrm{p}=0,060)$ e menor percentual de contrarreferência pela equipe especializada $(\mathrm{p}=0,028)$. A taxa de concordância diagnóstica global foi semelhante entre os dois modelos de UBS, com razoável nível de concordância (índice kappa de 44,5 e 43,0\%, respectivamente, para UBS com e sem equipe PSF).

Conclusão: A UBS com equipe de PSF não apresentou resultados compatíveis com o que seria de se esperar, em função de sua hipotética melhor qualidade de estrutura.

Descritores: Saúde mental, atenção básica a saúde, avaliação de serviços de saúde, Programa Saúde da Família.

\begin{abstract}
Introduction: The objective of this study was to compare the profile of mental health assistance provided at primary care units (PCUs) with and without a Family Health Program (FHP) team.

Methods: Observational study evaluating patients referred by PCUs located in the coverage area of a specialized mental health institution between April 2003 and March 2006.

Results: The PCU with a FHP team presented better global standards for data recording, higher exclusive participation of medical doctors on their referral to specialists $(p=0.000)$, lower capacity of patient retention $(p=0.099)$, higher rates of treatment dropout in secondary level $(p=0.060)$, and lower percentage of counter-referral by the specialized team $(p=0.028)$. The overall index of diagnostic agreement was similar for both types of PCU model, with a reasonable level of agreement (kappa index of 44.5 and $43.0 \%$, respectively, for PCUs with and without a FHP team). Conclusion: The PCU with a FHP team did not present results compatible with what would be expected based on its hypothetically better quality. Keywords: Mental health, primary health care, health services evaluation, Family Health Program.
\end{abstract}

\section{Correspondência:}

Prof. Dr. Mário Sérgio Ribeiro, Rua Severino Meireles, 325/902, CEP 38025-040, Juiz de Fora, MG. E-mail: mariosribeiro@acessa.com FAPEMIG: Projeto EDT 3322/06. As acadêmicas receberam bolsas do SUS-JF, UFJF e FAPEMIG.

Não há conflitos de interesse associados à publicação deste artigo.

Copyright $($ C Revista de Psiquiatria do Rio Grande do Sul - APRS 


\section{Introdução}

Assiste-se, em nosso país, a um sempre renovado e retomado processo de mudanças no rumo da assistência aos portadores de transtornos mentais: mudam-se as instituições - ou, ao menos, mudam-se seus nomes -, mudam-se as políticas assistenciais, mudam-se as teorias e mudam-se as propostas técnicas. Cabe-nos, todavia, questionar se a assistência efetivamente prestada tem-se transformado concretamente ${ }^{1}$.

O Relatório sobre a Saúde no Mundo ${ }^{2}$, especificamente focado na saúde mental, ressalta que o controle e o tratamento dos transtornos mentais, no contexto da Atenção Básica de Saúde (ABS), são fundamentais para que: um maior número de pessoas tenha acesso facilitado aos serviços de saúde; melhore a qualidade da atenção; sejam reduzidos os desperdícios financeiros por investigações diagnósticas desnecessárias e tratamentos inespecíficos ou inapropriados.

Embora existam evidências de que os transtornos mentais de menor gravidade possam ser mais efetivamente cuidados na atenção primária, muitos clínicos gerais e possivelmente alguns pacientes preferem a referência para equipes de saúde mental $^{3}$. Já há 10 anos, Kapczinski et al. ${ }^{4}$ indicavam que o problema central da prestação de serviços gerais de saúde em nosso país residiria em que esses serviços não estariam atendendo ao propósito de se elevar o nível da saúde física, mental e social da população. Ainda que se possa considerar que todo problema de saúde é sempre um problema de saúde mental ${ }^{5} \mathrm{e}$ que toda ação de saúde mental seja também de produção de saúde ${ }^{6}$, a situação descrita por Kapczinski et al. ${ }^{4}$, a rigor, não parece ter mudado substancialmente nos dias de hoje.

O Programa de Saúde da Família (PSF), implantado pelo Ministério da Saúde a partir de 1995, seguiu o sucesso do Programa de Agentes Comunitários de Saúde, implementado ainda em 1991. O foco das ações desses programas está na família e não no indivíduo, e seus profissionais devem buscar ativamente a população sob sua responsabilidade ${ }^{7,8}$. Desde 1996, o PSF se tornou um instrumento de reestruturação do Sistema Único de Saúde (SUS), contribuindo para uma mudança de paradigma da prática assistencial até então dominante ${ }^{9}$.

Estudo realizado no interior de Minas Gerais constatou que, dentre sujeitos apontados por profissionais de equipes do PSF como indivíduos com possíveis dificuldades psicológicas, $37,8 \%$ apresentavam evidências de transtornos mentais nãopsicóticos ${ }^{10}$. Da mesma forma, inquérito diagnóstico realizado com pacientes atendidos por equipes do PSF do município de Petrópolis identificou altas prevalências de transtornos mentais comuns - caracterizados por queixas depressivas e ansiosas inespecíficas - na população atendida por tais equipes: $56 \%$ dos sujeitos avaliados apresentariam algum transtorno mental comum e, desses, cerca de $33 \%$ poderiam apresentar um transtorno mental de maior gravidade ${ }^{11}$.

Tais resultados reforçam a necessidade de que equipes de ABS - em especial do PSF - estejam capacitadas para o atendimento de pessoas com transtornos mentais de menor gravidade, para além do habitual encaminhamento para serviços especializados ${ }^{10,12}$.

Todavia, na prática ainda se identifica baixa efetividade no atendimento a pacientes com queixas na área da saúde mental no nível primário de sistemas de saúde. Um dos fatores que reduz a qualidade desse atendimento é a incapacidade dos profissionais de diagnosticar corretamente e de cuidar das patologias mentais presentes na sua clientela habitual. A presença de sofrimento mental nos pacientes atendidos na rede básica de saúde costuma passar despercebida, apesar de sua alta prevalência ${ }^{11,13,14}$.

O modelo de consultoria-ligação ${ }^{3}$ seria um modelo de interface entre o cuidado primário e o especializado que dá grande ênfase ao desenvolvimento de ligações próximas entre a equipe de ABS e de especialistas em saúde mental, objetivando: a) reduzir o número de referências de tratamento de transtornos leves; b) encorajar a referência de transtornos mentais graves; e c) aumentar a competência dos generalistas na detecção e manejo dos problemas mentais. Segundo Bower $\&$ Gask $^{15}$, esse modelo apresentaria vantagens significativas em relação a outras formas de organização do cuidado à saúde mental e já teria sido avaliado como bastante eficaz no trabalho em atenção primária à saúde mental.

Adotando a perspectiva de consultoria-ligação, iniciou-se no município de Juiz de Fora (MG), em 1997, um processo de reformulação do modelo até então adotado na atenção à saúde mental. O Sistema Municipal de Saúde Mental de Juiz de Fora (SMSM-JF) concentrou-se, fundamentalmente, na inserção do subsetor de saúde mental no sistema regionalizado, descentralizado e hierarquizado, estendendo as ações de saúde mental às UBS do município ${ }^{16}$.

O SMSM-JF comporta 11 diferentes Centros Regionais de Referência em Saúde Mental (CRRESAM), cada um atendendo um grupo das 41 UBS de determinada área da cidade de Juiz de Fora. O CRRESAM-Oeste, objeto desta avaliação, foi responsável pelos pacientes referenciados por cinco unidades básicas de saúde (UBS) da região conhecida como "cidade alta". No SMSM-JF, as UBS devem realizar a avaliação inicial dos pacientes de acordo com instrumentos e orientações de conduta propostas em manual desenvolvido para este fim - os Protocolos de Conduta do SMSM-JF ${ }^{17}$. Caso necessário - em virtude da complexidade do caso -, os profissionais dessas unidades referem o paciente para a equipe especializada responsável pelo atendimento dos pacientes e supervisão das equipes de sua área de abrangência, realizando treinamento e educação permanente dos técnicos das UBS. Todos os profissionais de saúde das UBS da área de referência do CRRESAM-Oeste, com e sem equipes de PSF, foram preliminarmente capacitados e as equipes de cada UBS eram regularmente supervisionadas por técnicos do serviço secundário de referência com vistas ao aperfeiçoamento contínuo do processo assistencial. Ressalta-se que, nesse modelo assistencial, espera-se que as UBS encaminhem ao nível secundário os pacientes mais graves, que estejam motivados e tenham disponibilidade para o tratamento mais intensivo com a equipe especializada. 
Ao final da avaliação pela equipe especializada, seus profissionais podem contrarreferenciar os pacientes para acompanhamento nas UBS ou referenciá-los a programas especiais de atendimento a grupos específicos ${ }^{18}$. Nesse sistema, as novas tarefas desenvolvidas pelos profissionais do nível secundário, bem como a mudança de estilo de prática especializada exigida de seus executores, são uma expressão concreta da necessária mudança de padrão de trabalho apontada por diferentes autores que se dedicam ao tema da atenção primária à saúde mental ${ }^{19,20}$.

O objetivo deste estudo é comparar o perfil de trabalho em saúde mental realizado por duas formas de organização do trabalho em ABS - o modelo PSF, aqui representado por uma das UBS e o modelo tradicional, não-PSF, representado por quatro UBS. Todas UBS estudadas estavam vinculadas ao mesmo serviço de referência e seus profissionais (médicos, enfermeiros e assistentes sociais) foram igualmente capacitados para o trabalho no SMSM-JF.

Enquanto as quatro UBS do modelo tradicional participaram do projeto piloto de implantação do SMSM-JF, ainda em 1997, a UBS com PSF iniciou seu funcionamento em 2000, já com equipe de PSF. Visando avaliar a qualidade e a efetividade do processo assistencial ${ }^{21,22}$, estudou-se, especificamente: a capacidade de retenção dos pacientes na ABS; a qualidade dos registros; a integração das equipes das UBS; a concordância entre hipóteses diagnósticas dos profissionais da atenção básica e diagnósticos efetuados pelos profissionais do nível secundário; e o abandono ou permanência dos pacientes em tratamento.

\section{Método}

\section{Desenho e critérios de inclusão}

Partiu-se de estudo observacional, sem intervenção do grupo de pesquisadores na alocação dos sujeitos ou procedimentos terapêuticos. Foram avaliados os registros de todos os pacientes encaminhados pelas UBS da área de abrangência do nível secundário em três períodos distintos - abril de 2003 a março de 2004, abril de 2004 a março de 2005 e abril de 2005 a março de 2006 - e que compareceram a pelo menos uma consulta com a equipe de especialistas, independente da idade, num total de 1.397 sujeitos. Não foram incluídos 15 pacientes encaminhados para reavaliação ainda dentro de cada um dos três períodos considerados.

\section{Variáveis e parâmetros}

As características avaliadas constam dos prontuários clínicos semiestruturados que circulam no SMSM-JF, bem como dos registros efetuados ao longo do atendimento secundário pela equipe de pesquisa. A digitalização do banco de dados e a análise dos resultados foram realizadas por meio dos programas Epi-Info 6.04d e Statistical Package for the
Social Sciences for Windows (SPSS) versão 14.0 (licença de uso CFOP 9656438).

Os diagnósticos principais foram estabelecidos pelos especialistas, de acordo com a Classificação Internacional de Doenças (CID-10) ${ }^{23}$, apenas agrupando os diagnósticos entre F50 a F69 como categoria única de "outros transtornos psiquiátricos" e entre F80 a F99 como "transtornos mentais da criança ou do adolescente". As hipóteses diagnósticas efetuadas pelos técnicos das UBS devem ser informadas na ficha semiestruturada de referência. Reitera-se que os técnicos de todas as categorias profissionais do nível primário foram capacitados para realizar hipóteses diagnósticas psiquiátricas básicas, com a utilização dos protocolos de conduta do SMSM$\mathrm{JF}^{17}$. Para adequar-se ao padrão assistencial estabelecido nesse protocolo assistencial, a variável "situação de tratamento" do paciente - isto, é sua localização no sistema assistencial - foi avaliada 2 meses após cada período estudado.

Para a avaliação da qualidade da referência ao nível secundário, foram utilizados cinco parâmetros: 1) o percentual de pacientes referenciados em relação ao total de ações básicas efetivamente realizadas por cada UBS; 2) o percentual de nãoregistro de dados pelos profissionais da ABS; 3 ) o percentual de encaminhamentos sob responsabilidade de cada categoria profissional dos técnicos da equipe básica de saúde; e 4) as taxas de concordância entre a hipótese diagnóstica efetuada pelos técnicos das UBS e o diagnóstico indicado pelos profissionais do nível secundário; e 5) a variável "situação de tratamento", pela qual se apresenta os quantitativos de pacientes que permaneceram em tratamento com a equipe especializada, que foram contrarreferenciados às UBS, referenciados a programas especiais ou que abandonaram o tratamento no nível secundário. Com esses parâmetros, estimou-se: a capacidade de retenção de usuários no serviço de saúde básica; a adequada realização dos registros; a integração da equipe das UBS na avaliação dos referenciados; a habilidade dos profissionais das UBS em identificar transtornos mentais (hipótese diagnóstica) e corretamente referenciar ao nível secundário, por meio de comparação com o diagnóstico estabelecido pela equipe de especialistas; e a taxas de abandono ou continuidade de tratamento.

A variável "responsável pelo encaminhamento" foi preenchida considerando-se as três categorias profissionais da ABS (médica, enfermagem e serviço social) que se responsabilizaram - apondo sua assinatura ao prontuário semiestruturado - pela referência de cada paciente.

\section{Análise estatística}

Para a caracterização dos pacientes (Tabela 1), estão indicados, para cada variável, os percentuais relativos à categoria na qual o grupo da UBS com equipe de PSF apresentou taxas de respostas mais elevadas, considerados os registros efetivamente realizados. Entre parênteses estão explicitados os percentuais de não-registros da característica avaliada em relação ao total de sujeitos avaliados. 
Tabela 1 - Características demográficas, econômicas e ocupacionais de pacientes referenciados ao nível secundário de UBS com equipe de PSF e sem equipe de PSF*

\begin{tabular}{|c|c|c|c|}
\hline Variáveis & $\begin{array}{c}\text { UBS com PSF } \\
(n=261) \\
\%^{\dagger}(\% \text { faltante })^{\ddagger}\end{array}$ & $\begin{array}{c}\text { UBS sem PSF } \\
(n=1.136) \\
\%(\% \text { faltante }) \\
\end{array}$ & $\mathbf{p}$ \\
\hline \multicolumn{4}{|l|}{ Demográficas } \\
\hline Sexo masculino & $40,2(0)$ & $39,5(0)$ & 0,833 \\
\hline Cor branca & $70,2(28,0)$ & $69,8(30,5)$ & 0,919 \\
\hline Idade acima da média do grupo $(39,2$ anos; $\mathrm{DP}=15,8)$ & $56,3(6,1)$ & $54,3(8,2)$ & 0,569 \\
\hline Naturais de outras cidades que não Juiz de Fora & $42,3(35,6)$ & $41,2(31,8)$ & 0,792 \\
\hline \multicolumn{4}{|l|}{ Sociais } \\
\hline Baixa escolaridade ${ }^{\S}$ & $81,2(28,7)$ & $78,5(25,9)$ & 0,416 \\
\hline Casados/em união conjugal estável & $47,2(16,5)$ & $47,1(20,5)$ & 0,961 \\
\hline Se casado, não é o primeiro relacionamento conjugal & $32,4(87,0)$ & $23,5(65,8)$ & 0,245 \\
\hline Não têm filhos & $29,5(29,9)$ & $28,2(29,7)$ & 0,715 \\
\hline $\begin{array}{l}\text { Moradores na residência acima da média do } \\
\text { grupo }(3,9 \text { pessoas; } \mathrm{DP}=1,7)\end{array}$ & $64,9(22,6)$ & $57,0(25,9)$ & 0,042 \\
\hline Professam outra religião que não a católica & $32,1(36,8)$ & $26,9(32,8)$ & 0,172 \\
\hline Frequentam o culto & $71,3(45,2)$ & $66,2(38,7)$ & 0,237 \\
\hline \multicolumn{4}{|l|}{ Ocupacionais } \\
\hline Profissão vinculada a comércio e serviços & $55,1(43,7)$ & $45,0(44,1)$ & 0,027 \\
\hline Não trabalham na profissão & $69,2(44,1)$ & $50,6(51,3)$ & 0,000 \\
\hline Desempregados & $47,7(41,4)$ & $38,1(49,4)$ & 0,030 \\
\hline \multicolumn{4}{|l|}{ Econômicas } \\
\hline Não dependem economicamente de outrem & $38,2(28,7)$ & $31,1(30,4)$ & 0,063 \\
\hline Não são arrimo de família & $82,2(37,5)$ & $68,9(40,9)$ & 0,000 \\
\hline Renda pessoal menor que um SM & $76,8(37,2)$ & $66,9(37,0)$ & 0,013 \\
\hline Renda familiar menor ou igual a um SM & $55,2(36,8)$ & $44,7(38,9)$ & 0,015 \\
\hline
\end{tabular}

CRRESAM = Centros Regionais de Referência em Saúde Mental; DP = desvio padrão; PSF = Programa de Saúde da Família;

$\mathrm{SM}=$ salário mínimo; UBS = unidade básica de saúde.

* Dados constantes da ficha de encaminhamento UBS-CRRESAM.

† Percentual relativo aos dados efetivamente registrados.

* Percentual de não-registro da variável pela UBS.

$\S$ Analfabeto até ensino fundamental completo.

Para a efetivação dos procedimentos de cruzamento de dados (tabelas de contingência), as variáveis foram reagrupadas em duas categorias. Para tanto se utilizou de: 1) categorias "acima" ou "abaixo" da média, para variáveis quantitativas; e 2) redução das categorias a duas possibilidades opostas - tais como professar ou não professar a religião católica -, no caso de variáveis categóricas. Os sujeitos sem o efetivo registro em cada variável (casos missing), não foram considerados para a realização das tabelas de contingência. $O$ teste do qui-quadrado de Pearson foi utilizado para avaliar a significância dos resultados encontrados nos cruzamentos de dados relativos à UBS com equipe de PSF em relação aos dados das UBS sem PSF, tomadas em conjunto. Os valores de $\mathrm{p}$ foram considerados estatisticamente significantes quando $\leq 0,05$ e marginalmente significantes quando $>0,05$ até 0,1 . Nos quadros estão apresentadas as variáveis reagrupadas e nas tabelas as variáveis que não o foram; neste caso, o qui-quadrado foi calculado tomando-se cada categoria em relação ao conjunto das demais.
O índice kappa foi utilizado para verificar a concordância entre a hipótese diagnóstica realizada pelos técnicos das UBS e o diagnóstico realizado pelos profissionais do nível secundário. Valores de kappa entre 100 e $80 \%$ são considerados ótimos; entre 80 e $60 \%$, bons; entre 60 e $40 \%$, regulares; entre 40 e $20 \%$, ruins; e abaixo de $20 \%$, péssimos ${ }^{24}$. Entre abril de 2003 e março de 2004, não foram devidamente diferenciados os pacientes que abandonaram o tratamento daqueles contrarreferenciados, não tendo sido possível avaliar a categoria abandono quanto a esse período.

\section{Aspectos éticos}

Esta pesquisa foi aprovada pelo Comitê de Ética em Pesquisa (CEP) do Hospital Universitário do Universidade Federal de Juiz de Fora (UFJF), processo no 377.062.2004, e pelo CEP da UFJF (Parecer 193/2007) e cumpre os princípios éticos contidos na Declaração de Helsinki. 


\section{Resultados}

Observa-se elevados percentuais de dados não-registrados, para ambos os tipos de organização do trabalho em ABS estudados. Foram referenciados mais pacientes do sexo feminino, que se declararam como de cor branca, católicos e naturais de Juiz de Fora. A idade média foi de 39,2 anos. Houve predomínio de sujeitos solteiros ou sem união estável, com filhos, em cuja residência moravam mais de três pessoas, que trabalhavam no setor de comércio e serviços, desempregados e com baixa renda familiar e pessoal. A maioria dos sujeitos dependia economicamente de outrem e não era arrimo de família.

O número de pacientes referenciados que efetivamente compareceram ao serviço secundário de saúde mental foi avaliado em relação ao número de ações básicas de saúde efetivamente realizadas (Tabela 2). Evidencia-se que o número de pacientes referenciados e que compareceram ao atendimento especializado em saúde mental é bastante pequeno em relação ao número de ações básicas de saúde efetivamente realizadas nas UBS. Deve-se considerar que, apesar de cada referência efetivada indicar um paciente específico, o total de ações básicas não se refere ao número de diferentes usuários atendidos pelas UBS. Percebe-se que a UBS com equipe de PSF realizou um percentual de referências mais elevado que o das UBS com equipe tradicional. Ainda que os percentuais de referências tenham sido semelhantes $-0,78$ e $0,70 \%$ para, respectivamente, UBS com e sem equipe de PSF -, o elevado número de sujeitos estudados permitiu que se identificasse diferença marginalmente significante entre os dois tipos estudados $(\mathrm{p}=0,099)$.

Tabela 2 - Referências ao nível secundário

\begin{tabular}{|c|c|c|c|c|c|c|}
\hline \multirow{2}{*}{$\begin{array}{l}\text { Referências ao } \\
\text { nível secundário }\end{array}$} & \multicolumn{2}{|c|}{ UBS com PSF } & \multicolumn{2}{|c|}{ UBS sem PSF } & \multicolumn{2}{|l|}{ Total } \\
\hline & Frequência & $\%$ & Frequência & $\%$ & Frequência & $\%$ \\
\hline $\begin{array}{l}\text { Consultas que geraram referências } \\
\text { que efetivamente compareceram } \\
\text { no nível secundário }\end{array}$ & 261 & 0,78 & 1.136 & 0,70 & 1.397 & 0,71 \\
\hline $\begin{array}{l}\text { Consultas que não geraram referências } \\
\text { que efetivamente compareceram no } \\
\text { nível secundário }\end{array}$ & 33.225 & 99,22 & 161.971 & 99,30 & 195.196 & 99,29 \\
\hline $\begin{array}{l}\text { Ações básicas de saúde efetivamente } \\
\text { realizadas* }\end{array}$ & 33.486 & 100 & 163.107 & 100 & 196.593 & 100 \\
\hline
\end{tabular}

As taxas de pacientes referenciados sem a devida identificação do "responsável pelo encaminhamento" foi de $17,9 \%$ para as UBS tradicionais e de $11,5 \%$ para a UBS com PSF ( $p=0,009)$. Na Tabela 3, observa-se que os percentuais variaram de forma estatisticamente significante para todas as categorias profissionais: a UBS com equipe de PSF foi a que mais encaminhou pacientes sob responsabilidade apenas do médico, enquanto as UBS tradicionais encaminharam o maior percentual de pacientes com a assinatura de mais de um profissional da equipe.

Tabela 3 - Responsável pelo encaminhamento*

\begin{tabular}{lccc}
\hline & $\begin{array}{c}\text { UBS com PSF } \\
\mathbf{\%}(\mathbf{n}=\mathbf{2 3 1})\end{array}$ & $\begin{array}{c}\text { UBS sem PSF } \\
\mathbf{\%}(\mathbf{n}=\mathbf{9 3 3})\end{array}$ & $\mathbf{p}^{\dagger}$ \\
Responsável pelo encaminhamento & 48,5 & 23,6 & 0,000 \\
Somente médico & 6,9 & 20,6 & 0,000 \\
Somente enfermeiro & 6,5 & 3,5 & 0,043 \\
Somente assistente social & 38,1 & 52,3 & 0,000 \\
Mais de um responsável & 100 & 100 & - \\
Total & &
\end{tabular}

PSF $=$ Programa de Saúde da Família; UBS = unidade básica de saúde

* Não considerados os pacientes sem identificação do responsável.

† Relativo à comparação de cada categoria ao conjunto das demais. 
O registro de "hipótese diagnóstica" foi maior na UBS com PSF que nas UBS tradicionais - respectivamente, 73,90 e $65,8 \%(p=0,013)$. Para o "diagnóstico principal", dado pela equipe de especialistas, observou-se não ter havido registro em apenas 6,0\% dos casos (Tabela 4). Quanto às hipóteses diagnósticas, apenas para os transtornos mentais orgânicos se observou uma diferença marginalmente significante $(\mathrm{p}=$ 0,072 ) entre os dois modelos de UBS. Para o diagnóstico do nível secundário, diferença marginalmente significante foi observada para o grupo dos retardos mentais $(\mathrm{p}=0,053)$.

Tabela 4 - Registro de hipótese diagnóstica nas UBS e diagnóstico psiquiátrico pela equipe especializada*

\begin{tabular}{lccc}
\hline Variáveis & UBS com PSF (\%) & UBS sem PSF (\%) & $\mathbf{p}^{\dagger}$ \\
\hline Hipótese diagnóstica pela equipe da UBS & $(\mathrm{n}=193)$ & $(\mathrm{n}=747)$ & 0,072 \\
Transtorno mental orgânico & 9,3 & 5,8 & 0,252 \\
Transtorno por uso de substância psicoativa & 12,4 & 9,6 & 0,400 \\
Transtorno psicótico/delirante & 9,3 & 7,5 & 0,820 \\
Transtorno de humor & 29,0 & 29,9 & 0,128 \\
Transtorno de ansiedade & 36,3 & 42,3 & 0,180 \\
Transtorno de personalidade & 0,5 & 1,9 & 0,982 \\
Mais de uma hipótese diagnóstica & 3,1 & 3,1 & - \\
Total & 100 & 100 & 0,306 \\
Diagnóstico psiquiátrico pela equipe especializada & $(\mathrm{n}=245)$ & $(\mathrm{n}=1.068)$ & 0,261 \\
Transtorno mental orgânico & 5,7 & 4,2 & 0,206 \\
Transtorno por uso de substância psicoativa & 12,2 & 9,8 & 0,472 \\
Psicoses e esquizofrenia & 2,9 & 4,7 & 0,397 \\
Transtorno de humor & 23,3 & 25,5 & 0,053 \\
Transtorno de ansiedade & 34,3 & 37,2 & 0,326 \\
Retardo mental & 11,0 & 7,3 & 0,153 \\
Transtorno mental da criança e do adolescente & 6,9 & 5,3 & - \\
Outros transtornos psiquiátricos & 3,7 & 6,0 & 100 \\
Total & 100 & & \\
\hline
\end{tabular}

PSF = Programa de Saúde da Família; UBS = unidade básica de saúde.

* Não considerados os pacientes sem registro de hipótese diagnóstica.

${ }^{\dagger}$ Relativo à comparação de cada categoria ao conjunto das demais.

A Tabela 5 explicita os níveis de concordância entre hipótese diagnóstica e diagnóstico principal. Observa-se que a hipótese de transtorno por uso de substância psicoativa foi a que resultou em melhores taxas de concordância: coeficiente kappa $^{24}$ de 78,7 e 81,9\%, atingindo nível excelente na UBS com equipe de PSF. A taxa de concordância global foi semelhante entre os dois modelos de UBS, com razoável nível de concordância (44,5 e 43,0\%); entretanto, para os quadros psicóticos, a taxa de concordância observada para a UBS com equipe de PSF foi muito mais baixa que para as UBS sem equipe de PSF (14,0 e 31,5\%, respectivamente). Não foi possível calcular o coeficiente kappa para os transtornos de personalidade para a UBS com equipe de PSF; para as UBS tradicionais, a concordância foi desprezível $(8 \%)$.

A Tabela 6 indica a localização dos pacientes dentro do sistema de saúde mental após avaliação no nível secundário, ou seja, a quantidade de pacientes que abandonaram o tratamento no nível secundário, que permaneceram em tratamento nesse mesmo nível, foram contrarreferenciados às UBS ou referenciados a algum dos programas especiais. De forma marginalmente significativa $(p=0,060)$, os abandonos de tratamento no nível secundário foram mais frequentes entre pacientes da UBS com equipe de PSF, enquanto as contrarreferências para continuidade de tratamento no nível primário foram significantemente mais elevadas para as UBS tradicionais $(\mathrm{p}=0,028)$. Percentuais quase idênticos de pacientes dos dois modelos de UBS permaneceram em tratamento no nível secundário ou foram referenciados aos programas superespecializados.

\section{Discussão}

\section{Aspectos sociodemográficos e econômicos}

Os dois subgrupos foram semelhantes quanto aos aspectos sociodemográficos; a única exceção foi ter sido registrado um número significantemente mais elevado de residências com mais de três moradores $(p=0,042)$ dentre os pacientes da UBS com equipe de PSF. Todavia, os aspectos ocupacionais e econômicos discriminaram os dois grupos: de forma significante, 
Tabela 5 - Concordância entre hipótese diagnóstica pela UBS

e diagnóstico principal registrado pelos profissionais do nível secundário*

\begin{tabular}{|c|c|c|c|c|c|c|c|c|}
\hline \multirow{2}{*}{$\begin{array}{l}\text { Hipótese } \\
\text { diagnóstica }\end{array}$} & \multirow[b]{2}{*}{ TMO } & \multirow[b]{2}{*}{ TUSPA } & \multicolumn{4}{|c|}{ Diagnóstico principal } & \multirow[b]{2}{*}{ Total } & \multirow[b]{2}{*}{ kappa (\%) } \\
\hline & & & Esquizofrenia $^{\dagger}$ & TH & TA & $\mathbf{T P}$ & & \\
\hline \multicolumn{9}{|c|}{ UBS com equipe PSF } \\
\hline TMO & 3 & 0 & 1 & 0 & 2 & 7 & 13 & 22,0 \\
\hline TUSPA & 1 & 19 & 1 & 1 & 1 & 0 & 23 & 81,9 \\
\hline Psicóticos & 2 & 2 & 2 & 4 & 2 & 5 & 17 & 14,0 \\
\hline $\mathrm{TH}$ & 2 & 0 & 0 & 23 & 17 & 3 & 45 & 33,9 \\
\hline TA & 1 & 1 & 1 & 15 & 38 & 4 & 60 & 40,9 \\
\hline $\mathrm{TP}$ & - & - & - & - & - & - & - & $-\$$ \\
\hline Total & 9 & 22 & 5 & 43 & 60 & 19 & 158 & $44,5^{\S}$ \\
\hline \multicolumn{9}{|c|}{ UBS sem equipe PSF } \\
\hline TMO & 7 & 1 & 3 & 6 & 1 & 16 & 34 & 19,9 \\
\hline TUSPA & 2 & 51 & 0 & 3 & 6 & 0 & 62 & 78,7 \\
\hline Psicóticos & 7 & 0 & 13 & 14 & 7 & 10 & 51 & 31,5 \\
\hline $\mathrm{TH}$ & 4 & 6 & 4 & 91 & 63 & 8 & 176 & 29,9 \\
\hline TA & 5 & 5 & 3 & 67 & 183 & 9 & 272 & 43,1 \\
\hline $\mathrm{TP}$ & 0 & 1 & 0 & 2 & 4 & 3 & 10 & 8,0 \\
\hline Total & 25 & 64 & 23 & 183 & 264 & 46 & 605 & $43,0^{\S}$ \\
\hline
\end{tabular}

PSF = Programa de Saúde da Família; TA = transtorno de ansiedade; TH = transtorno de humor; TMO = transtorno mental orgânico; $\mathrm{TP}=$ transtorno de personalidade; TUSPA = transtorno por uso de substância psicoativa; UBS = unidade básica de saúde.

* Considerados apenas os pacientes com registro de hipótese diagnóstica e diagnóstico.

$\dagger$ Esquizofrenia ou do grupo F20 a F29.

* O coeficiente kappa não foi calculado pela inexistência de, ao menos, uma concordância diagnóstica.

$\S$ O kappa total exclui a hipótese diagnóstica e o diagnóstico de transtorno de personalidade.

Tabela 6 - Localização dos pacientes nos dispositivos assistenciais disponíveis de pacientes referenciados ao nível secundário*

\begin{tabular}{lccc}
\hline Situação de tratamento ${ }^{* \dagger}$ & $\begin{array}{c}\text { UBS com PSF } \\
\mathbf{\%}(\mathbf{n}=\mathbf{1 6 7})\end{array}$ & $\begin{array}{c}\text { UBS sem PSF } \\
\mathbf{\%}(\mathbf{n}=\mathbf{7 1 8})\end{array}$ & $\mathbf{p}^{\ddagger}$ \\
\hline Abandono & 32,9 & 25,8 & 0,060 \\
Contrarreferenciado UBS & 30,5 & 39,7 & 0,028 \\
Permanece com a equipe de referência secundária & 12,6 & 10,3 & 0,393 \\
Referenciado a programas superespecializados & 22,8 & 22,4 & 0,926 \\
Outros & 1,2 & 1,8 & 0,580 \\
Total & 100 & 100 & - \\
\hline
\end{tabular}

PSF = Programa de Saúde da Família; UBS = unidade básica de saúde.

* Foram excluídos todos os pacientes "sem registro" de dados $(1,2 \%)$ e também os 501 pacientes referenciados no primeiro período

estudado, quando abandono e contrarreferência não foram diferenciados.

† Avaliadas, respectivamente, em 31/05/2005 e 31/05/2006.

${ }^{*}$ Relativo à comparação de cada categoria ao conjunto das demais.

§ Inclui pacientes encaminhados para internação psiquiátrica, alta clínica e falecimento.

os pacientes da UBS com equipe de PSF apresentaram piores condições de trabalho e renda, ainda que, em maior percentual relativo, não dependam economicamente de outrem $(p=0,063)$. $\mathrm{O}$ não-registro de dados sociodemográficos, econômicos e ocupacionais foi igualmente elevado para ambos os modelos de UBS estudadas, não se identificando, porém, um padrão consistentemente predominante.

\section{Capacidade de retenção de usuários no serviço de saúde básica}

De acordo com dados do Boletim de Produção SIA-SUS (Sistema de Informação Ambulatorial do Sistema Único de Saúde) e SIAB (Sistema de Informação da Atenção Básica), 196.593 ações básicas de saúde foram efetivamente realizadas 
pelas UBS no período do estudo, enquanto que 1.397 pacientes foram referenciados e, de fato, compareceram à primeira consulta serviço secundário de saúde mental, correspondendo a $0,71 \%$ do total de ações básicas de saúde. Uma vez que cada referência efetivada refere-se a um usuário específico, enquanto que o número de ações básicas não reflete o total de diferentes usuários atendidos pelas UBS, tais valores devem ser tomados apenas como critério de comparação dos dois modelos organizacionais e não utilizados para avaliação das taxas de incidência e prevalência de transtornos mentais na população atendida. Percebe-se, portanto, que a UBS com equipe de PSF realizou um percentual de referências mais elevado que o das tradicionais. Apesar da semelhança dos percentuais de referências - 0,78 e $0,70 \%$ para, respectivamente, UBS com e sem equipe de PSF -, o elevado número de sujeitos estudados permitiu que se identificasse diferença marginalmente significante entre os dois modelos de UBS $(p=0,099)$. Ainda que a UBS com equipe de PSF tenha realizado um percentual de referências mais elevado que o das tradicionais, não se pode afirmar que esta UBS tenha encaminhado pacientes ao nível secundário de saúde mental de forma excessiva: sua equipe poderia estar identificando melhor os usuários necessitados de assistência especializada em saúde mental ou refletindo nesse encaminhamento uma maior prevalência de transtornos mentais entre seus usuários. Conforme indicado por Wang et al. ${ }^{25}$, as distintas características econômicas dos sujeitos podem influenciar a demanda por atendimento em saúde mental; todavia, uma discussão mais aprofundada dessa questão extrapola os limites do presente estudo.

\section{Realização dos registros pela equipe de ABS}

Apesar de ter sido elevado o percentual de não-registro dos dados relacionados ao profissional "responsável pelo encaminhamento" e da "hipótese diagnóstica", para os dois modelos de UBS consideradas, aquela com equipe de PSF apresentou resultados significantemente melhores: o percentual de ausência de registro do "responsável pelo encaminhamento" foi de 11,5\% para a UBS com PSF e de 17,9\% para o conjunto das demais (p $=0,009)$; o não-registro de "hipótese diagnóstica" foi de $26,1 \%$ para a UBS com PSF e de $34,2 \%$ para as UBS tradicionais (p $=0,013)$. Tais resultados poderiam, em princípio, sugerir tanto uma reduzida habilidade dos profissionais das UBS tradicionais em lidar com o diagnóstico de transtornos mentais quanto também uma precária adesão às normas de trabalho.

Tal desconsideração dos protocolos assistenciais do SMSM-JF parece refletir um padrão de trabalho de profissionais de ABS do município de Juiz de Fora: segundo Ronzani et al. ${ }^{26}$, uma certa "sobrecarga de trabalho" - conforme percebido e referido pelos técnicos da $\mathrm{ABS}$ - faria com que qualquer mudança na rotina assistencial tenda sofrer resistência da equipe, ao ser interpretada apenas como uma nova atividade a ser desenvolvida. Ainda que o registro desses dados tenha sido claramente normatizado no SMSM-JF, deve-se ter em conta que o registro de ações de saúde mental não se inclui entre as exigências do Ministério da Saúde na avaliação do trabalho dos serviços de ABS, o que pode contribuir para elevado percentual de não-registro de dados. A diferença encontrada é corroborada pelos resultados de pesquisa de Facchini et al. ${ }^{27}$ que indicaram que as UBS com equipes de PSF utilizavam protocolos específicos em suas atividades assistenciais com maior frequência que as tradicionais.

Recente estudo publicado por Weinmann et al. ${ }^{28}$ evidenciou que a não-utilização de protocolos clínicos é bastante comum e generalizada. Segundo os autores, a simples distribuição de protocolos não é suficiente para sua implementação: mesmo que os clínicos os conheçam, eles não os aplicam necessariamente. Segundo Leucht ${ }^{29}$, o trabalho de Weinmann et al. ${ }^{28}$ não deve ser considerado uma "palavra final" sobre o tema, importando identificar as razões para tal resistência. Existem, todavia, evidências empíricas de que a adequada utilização de protocolos assistenciais contribui para a melhoria da qualidade da assistência prestada também na área da saúde mental ${ }^{30}$.

\section{Integração da equipe das UBS na avaliação dos pacientes encaminhados}

Os resultados relativos à responsabilidade pelos encaminhamentos poderiam indicar que as UBS tradicionais funcionariam de forma mais integrada; por outro lado, poderiam também significar maior participação dos médicos que atuam no PSF na rotina assistencial da UBS. Deve-se levar em consideração que a UBS com equipe de PSF iniciou seu funcionamento em 2000 - mais recentemente que as demais, que participaram do projeto piloto de implantação do SMSMJF ainda em 1997 -, sem que sua equipe estivesse plenamente constituída. A possibilidade de que uma falta de entrosamento de seus componentes possa ter contribuído para os resultados encontrados é coerente com as evidências de que - ao menos pelo fato de envolver uma questão de comunicação entre profissionais de diferentes formações - um eficiente trabalho de equipe não é fácil de ser atingido ${ }^{21}$.

\section{Habilidade dos profissionais das UBS em identificar transtornos mentais}

Entre os pacientes encaminhados, a "hipótese diagnóstica" mais frequente foi a de transtorno de ansiedade, consistente com o diagnóstico principal efetuado pela equipe de especialistas e com a mais elevada prevalência desses transtornos entre a população geral ${ }^{11,31}$. O percentual significantemente maior de pacientes com hipótese diagnóstica de transtornos mentais orgânicos referenciado pela UBS com equipe PSF pode indicar mais alta prevalência desses transtornos na área de abrangência desta UBS. Todavia, considerando-se o fato de que a hipótese diagnóstica de oligofrenia não consta dos prontuários clínicos semiestruturados do SMSM-JF, pode-se supor que pacientes com tal diagnóstico tenham sido encaminhados sob a rubrica de transtornos mentais orgânicos. Os resultados da avaliação da concordância entre hipótese diagnóstica e diagnóstico principal reforçam esta hipótese: para os transtornos mentais 
orgânicos, identifica-se maior percentual de pacientes não registrados nas interações relativas à UBS com equipe de PSF que às UBS sem equipe PSF - respectivamente quatro em 13 pacientes $(30,8 \%)$ e nove em 34 pacientes $(26,5 \%)$.

Observou-se que a hipótese de transtorno por uso de substância psicoativa foi a que resultou em melhores taxas de concordância diagnóstica. É conhecido um comportamento ambivalente dos profissionais da ABS com relação a tais transtornos: por um lado, baixa sensibilidade para demanda não-específica - isto é, pacientes com sinais e sintomas sem referência direta ao consumo de substâncias ${ }^{32}-\mathrm{e}$, por outro, excessiva sensibilidade para sintomatologia mental associada à referência explícita a uso de substâncias ${ }^{6}$.

A baixíssima concordância diagnóstica quanto aos transtornos de personalidade pode refletir tanto uma limitação conceitual dos técnicos das UBS como uma baixa caracterização e explicitação criteriológica desses transtornos. Os transtornos de personalidade foram historicamente vinculados aos conceitos de personalidade psicopática e psicopatia e, até hoje, costumam ser confundidos com psicose e tomados como sinônimo de doença mental ${ }^{33}$.

Já há algum tempo vêm sendo discutidas as dificuldades relativas à confiabilidade dos diagnósticos psiquiátricos, mesmo entre especialistas ${ }^{34}$. Mesmo após as mudanças processadas nos critérios diagnósticos nas últimas duas décadas, ainda se evidencia que a incapacidade dos médicos de corretamente diagnosticar e tratar as patologias mentais presentes na sua clientela habitual se constituiria em importante fator relacionado à baixa efetividade no atendimento a pacientes com agravos e/ou queixas na área da saúde mental no nível primário $^{5,14}$.

Quanto aos transtornos psicóticos - que, a rigor, devem ser rapidamente identificados e encaminhados para tratamento com especialistas -, os resultados aqui apresentados indicaram que a UBS com equipe de PSF identificou menor percentual de verdadeiros psicóticos (menor especificidade diagnóstica). Da mesma forma, deixou de identificar como psicóticos maior número relativo de pacientes (três em cinco contra $10 \mathrm{em} 23$ das UBS tradicionais) que assim foram diagnosticados pela equipe de especialistas (falso-negativos). Vale lembrar que, no caso dessas patologias de maior risco de evolução desfavorável, é sempre melhor excesso de cautela que deixar passar um caso de psicose sem diagnóstico; ou, em outras palavras, a sensibilidade é sempre preferível à especificidade. Os resultados indicam que, se os profissionais da UBS com equipe de PSF verdadeiramente se preocuparam em referenciar pacientes psicóticos, deixaram de reconhecê-los com maior frequência relativa.

\section{Avaliação do abandono ou continuidade de tratamento}

O fato de não se ter observado diferença significativa quanto aos percentuais de pacientes que permaneceram em tratamento com a equipe secundária ou foram encaminhados para programas superespecializados indica que as equipes dos dois modelos de organização da ABS estudados identificaram e referenciaram número relativo de pacientes com real necessidade de tratamento especializado bastante semelhante. De forma marginalmente significante, os abandonos de tratamento no nível secundário foram mais frequentes entre pacientes da UBS com equipe de PSF, enquanto que as contrarreferências para continuidade de tratamento no nível primário foram significantemente mais elevadas para pacientes das UBS sem equipe de PSF. Tomados em conjunto, esses dados sugerem que as UBS com equipes tradicionais referenciaram pacientes com maior motivação para atendimento especializado.

Ainda que não se tenha chegado a resultados definitivos, estudos realizados nos últimos 15 anos têm identificado diversos fatores que se associam estatisticamente ao não-comparecimento às consultas $\mathrm{e} / \mathrm{ou}$ ao abandono de tratamento em saúde mental, tanto em nível primário quanto secundário. Alguns estudos ${ }^{35-37}$ registraram taxas de abandono significantemente mais elevadas entre pacientes solteiros, enquanto que o contrário foi registrado por outros autores ${ }^{38,39}$. Pacientes mais jovens tiveram maiores taxas de abandono nos estudos de Young et al..$^{38}$, Edlund et al. ${ }^{40}$ e Rossi et al. ${ }^{37}$. Melo \& Guimarães ${ }^{41}$ encontraram taxas de abandono significantemente mais elevadas em pacientes que não recebiam passes de ônibus para a consulta com especialistas. Regra geral, tais autores analisaram poucas variáveis econômicas e registraram diferenças principalmente quanto a características demográficas. Outro estudo com o mesmo grupo de pacientes aqui abordados - abrangendo maior número de variáveis demográficas e econômicas - identificou taxas significantemente mais elevadas de abandono de tratamento entre pacientes desempregados; de forma marginalmente significante, também foi observada entre pacientes que não eram arrimo de família ou afirmaram renda menor que um salário mínimo ${ }^{42}$. Nos limites deste estudo, não é possível descartar a possibilidade de as diferenças econômicas observadas entre usuários terem contribuído para o maior nível de abandono de pacientes provenientes da UBS com equipe PSF, uma vez que, conforme acima ressaltado, tais usuários apresentavam piores condições de renda e trabalho. Além disso, deve-se considerar que os pacientes da área de referência da UBS com equipe PSF, diferentemente dos moradores das outras áreas, não dispunham de uma linha de transporte coletivo com ponto de parada próximo ao serviço secundário.

Dadas as altas taxas de abandono de tratamento frequentemente encontradas em programas de saúde mental, considera-se que podem ocorrer distorções importantes na avaliação desses serviços, caso as informações relativas aos pacientes que não se mantiveram em tratamento não sejam incluídas ${ }^{38}$. A rigor, considera-se inefetiva uma proposta assistencial rejeitada por seus usuários ${ }^{43}$. O elevado percentual de não-registro de dados por parte das equipes das UBS limita a fidedignidade das informações e sua análise; por sua vez, o registro de dados pela equipe de especialistas pode ser prejudicado por abandono precoce dos pacientes atendidos.

Motivação para o tratamento com especialistas ${ }^{39}$, temor do estigma $^{25}$, possível resistência ao tratamento com psiquiatras ${ }^{44}$, 
modalidade de tratamento utilizada ${ }^{40,42}$, e características clínicas $^{45}$ e evolutivas ${ }^{46}$, específicas de determinados transtornos mentais, devem ser corretamente avaliadas pelos profissionais do nível primário, uma vez que influenciam o comparecimento e a permanência em tratamento no nível secundário. Melo \& Guimarães ${ }^{41}$ evidenciaram que a busca de serviço especializado de saúde mental por demanda espontânea foi associada a maiores taxas de abandono de tratamento.

As comparações entre os diferentes grupos diagnósticos não gerou significância estatística na comparação entre os dois modelos de UBS. Efetivamente, padrões conclusivos de associação entre diagnóstico psiquiátrico e abandono de tratamento ainda não foram identificados por diferentes pesquisadores $^{36-38,44,45,47}$.

O modelo de consultoria-ligação adotado pelo SMSM$\mathrm{JF}$, ao valorizar a comunicação entre os profissionais e entre especialistas e equipe de ABS deveria resultar em redução do número de referências de tratamento de transtornos leves e encorajar a referência de transtornos mentais graves ${ }^{3}$. Segundo Bower \& Gask ${ }^{15}$ ainda haveria uma lacuna entre o que é demonstrado ser eficaz em circunstâncias de pesquisa e sua efetividade na prática de rotina, como também parece acontecer com propostas assistenciais em outras áreas da saúde coletiva ${ }^{21}$.

Dentre as limitações deste estudo - como de todos os que utilizam semelhante desenho - aponta-se a reduzida possibilidade de generalização dos resultados, em especial pelo fato de que a referência dos sujeitos ao serviço especializado obedeceu aos protocolos de conduta do SMSM-JF ${ }^{17}$, sem qualquer outro critério de seleção ou exclusão. Deve-se, no entanto, observar que as variáveis aqui utilizadas podem, com facilidade, ser reproduzidas em outros contextos assistenciais, o que facilita a replicação deste estudo. $\mathrm{O}$ elevado número de sujeitos avaliados - dificilmente encontrado em estudos brasileiros - assegura a confiabilidade de seus resultados. Ressalta-se, ainda, que estudos que trabalham com situações de "vida real" contribuem, objetivamente, para uma avaliação da efetividade das práticas em saúde coletiva ${ }^{48,49}$. Além do mais, considera-se que, pelo simples fato de acontecerem, podem contribuir para a melhoria da qualidade da assistência ou, pelo menos, para prevenir sua deterioração.

\section{Conclusão}

Os resultados aqui apresentados indicaram, portanto, que a UBS com equipe de PSF apresentou melhor padrão de registros de dados, maior participação exclusiva de médicos em suas referências ao nível especializado, menor capacidade de retenção dos usuários, maiores taxas de abandono e menor percentual de contrarreferência pela equipe especializada. Os dois modelos de UBS estudados apresentaram baixas taxas de concordância diagnóstica, com resultados mais erráticos tendo sido evidenciados para a UBS com equipe de PSF. Os resultados corroboram a hipótese de que a qualidade da estrutura não se correlaciona diretamente com a qualidade do processo ou dos resultados e reforçam a importância de sistemas estruturados em que o funcionamento da interface nível primário secundário esteja apoiado em um consistente processo de supervisão e educação permanente dos profissionais envolvidos.

\section{Referências}

1. Ribeiro MS, Teixeira LS. Por uma ética das práticas em saúde mental. J Bras Psiquiatr. 1997;46(6):331-7.

2. WHO/SUPPORT. Relatório Mundial da Saúde 2001. Genebra: World Health Organization; 2001. http://www.who.int/whr/2001/en/whr01_po.pdf. Acessado 05 jun 2006.

3. Gask L, Sibbald B, Creed F. Evaluating models of working and the interface between mental health services and primary care. Br J Psychiatry. 1997;170:6-11.

4. Kapczinski F, Quevedo J, Madruga M, Busnello JV, Busnello EDA. Psiquiatria social e comunitária. R Psiquiatr RS. 1998;20(3):69-76.

5. Prince M, Patel V, Saxena S, Maj M, Maselko J, Phillips MR, et al. No health without mental health. Global Mental Health. Lancet. 2007;370(9590):859-77.

6. Ribeiro MS. O uso funcional e disfuncional de substâncias psicoativas. In: Ribeiro MS, org. Ferramentas para descomplicar a atenção básica em saúde mental. Juiz de Fora: Universidade Federal de Juiz de Fora; 2007. p. 55-80.

7. Senna MCM. Eqüidade e política de saúde: algumas reflexões sobre o Programa Saúde da Família. Cad Saude Publica. 2002;18(Suplemento):203-11.

8. Viana ALD, Poz MRD. A reforma do sistema de saúde no Brasil e o Programa de Saúde da Família. PHYSIS (Rio J.). 2005;15(Supl):225-64.

9. Brasil, Ministério da Saúde. Saúde da Família: uma estratégia de organização dos serviços de saúde. Brasília: Secretaria de Assistência à Saúde, 1996.

10. Bandeira M, Freitas LC, Carvalho Filho JGT. Transtornos mentais comuns em usuários do Programa de Saúde da Família. J Bras Psiquiatr. 2007;56(1):41-7.

11. Fortes S, Villano LAB, Lopes CS. Perfil nosológico e prevalência de transtornos mentais comuns em pacientes atendidos em unidades do Programa de Saúde da Família (PSF) em Petrópolis, Rio de Janeiro. Rev Bras Psiquiatr. 2008;30(1):32-7.

12. Rosa WAG, Labate RC. A contribuição da saúde mental para o desenvolvimento do PSF. Rev Bras Enferm. 2003;56(3):230-5.

13 Mari JJ. Morbilidad psiquiátrica en centros de atención primaria. Boletín de la Oficina Sanitaria Panamericana. 1988;104(2):171-81.

14. Kates N, Craven M, Crustolo AM, Nikolaou L, Allen C. Integrating mental health services within primary care. A Canadian program. Gen Hosp Psychiatry. 1997;19(5):324-32.

15. Bower $P$, Gask $L$. The changing nature of consultation-liaison in primary care: bridging the gap between research and practice. Gen Hosp Psychiatry. 2002;24(2):63-70.

16. Ribeiro MS, Stroppa ALPC, Salomão Neto A, Bastos MO, Costa DMT. Reforma psiquiátrica e atenção primária à saúde: o processo de implementação do sistema municipal de saúde mental de Juiz de Fora. Revista APS. 2003;6(1):19-29.

17. Ribeiro MS. Protocolos de Conduta do Sistema Municipal de Saúde Mental de Juiz de Fora. Juiz de Fora: SUS; 2000.

18. Ribeiro MS, Heckert U. Universidade e o cuidado à saúde: o "caso" do Serviço de Psiquiatria e Psicologia Médica do HU-UFJF. Rev Saude Dist Fed. 2005;16(12):35-47.

19. Bland RC. Psychiatry and the burden of mental illness. Can J Psychiatry. 1998;43(8):801-10.

20. Jenkins R. Linking epidemiology and disability measurement with mental health service policy and planning. Epidemiol Psichiatr Soc. 1998;7(2):120-6.

21. Barbara S. Atenção primária: equilíbrio entre necessidades de saúde, serviços e tecnologias. Brasília: Ministério da Saúde; 2002.

22. Salvador-Carulla L, Haro JM, Ayuso-Mateos JL. A framework for evidence-based mental health care and policy. Acta Psychiatr Scand Suppl. 2006;(432):5-11.

23. Caetano D. Classificação de transtornos mentais e de comportamento da CID-10: descrições clínicas e diretrizes diagnósticas. Porto Alegre: Artmed. 1993.

24. Jekel JF, Elmore JG, Katz DL. Epidemiology, biostatistics and preventive medicine. Philadelphia: Saunders; 1996.

25. Wang PS, Aguilar-Gaxiola S, Alonso J, Angermeyer MC, Borges G, Bromet EJ, et al. Use of mental health services for anxiety, mood, and substance disorders in 17 countries in the WHO world mental health surveys. Lancet. 2007;370(9590):841-50.

26. Ronzani TM, Ribeiro MS, Amaral MB, Formigoni MLOS. Implantação de rotinas de rastreamento do uso de risco de álcool e de uma intervenção breve na atenção primária à saúde: dificuldades a serem superadas. Cad Saude Publica. 2005;21(3):852-61. 
27. Facchini LA, Piccini RX, Tomasi E, Thumé E, Silveira DS, Siqueira FV, et al. Desempenho do PSF no Sul e no Nordeste do Brasil: avaliação institucional e epidemiológica da atenção básica à saúde. Rev Bras Saude da Família. 2007;13:28-41.

28. Weinmann S, Koesters M, Becker T. Effects of implementation of psychiatric guidelines on provider performance and patient outcome: systematic review. Acta Psychiatr Scand. 2007;115(6):420-33.

29. Leucht S. Psychiatric treatment guidelines: doctors non-compliance or insufficient evidence? (Editorial). Acta Psychiatr Scand. 2007;115(6):417-9.

30. Smith JL, Rost KM, Nutting PA, Elliott CE. Resolving disparities in antidepressant treatment and quality-of-life outcomes between uninsured and insured primary care patients with depression. Med Care. 2001;39(9):910-22.

31. Almeida Filho N, Mari JJ, Coutinho E, França JF, Fernades JG, Andreoli SB, et al. Estudo multicêntrico de morbidade psiquiátrica em áreas urbanas brasileiras (Brasília, São Paulo, Porto Alegre). Rev ABP-APAL. 1992;14(3):93-104.

32. Ribeiro MS. A inserção da saúde mental na Atenção Básica de Saúde. In: Ribeiro MS, org. Ferramentas para descomplicar a atenção básica em saúde mental. Juiz de Fora: Universidade Federal de Juiz de Fora; 2007. p. 15-26.

33. Mendes Filho RB, Morana HCP. Transtornos específicos da personalidade: semiologia em psiquiatria forense. Psychiatry On-line Brazil. 2003;8(12). http:// www.polbr.med.br/ano03/artigo1203 b.php. Acessado 03 mar 2007.

34. Caetano R. Causas de baja confiabilidad del diagnóstico em psiquiatría: Una revisión critica. Acta Psiquiatr Psicol Am Lat. 1978;24:115-30.

35. Olfson M. Primary care patients who refuse specialized mental health services. Arch Intern Med. 1991;151(1):129-32.

36. Matas M, Staley D, Griffin W. A profile of the noncompliant patient: a thirty-month review of outpatient psychiatry referrals. Gen Hosp Psychiatry. 1992;14(2):124-30.

37. Rossi A, Amaddeo F, Bisoffi G, Ruggeri M, Thornicroft G, Tansella M. Dropping out of care: inappropriate terminations of contact with community-based psychiatric services. Br J Psychiatry. 2002;181:331-8.

38. Young AS, Grusky O, Jordan D, Belin TR. Routine outcome monitoring in a public mental health system: the impact of patients who leave care. Psychiatr Serv. 2000;51(1):85-91.
39. Gonzalez J, Williams JW Jr., Noël PH, Lee S. Adherence to mental health treatment in a primary care clinic. J Am Board Fam Pract. 2005;18(2):87-96.

40. Edlund MJ, Wang PS, Berglund PA, Katz SJ, Lin E, Kessler RC. Dropping out of mental health treatment: patterns and predictors among epidemiological survey respondents in the United States and Ontario. Am J Psychiatry. 2002;159(5):845-51.

41. Melo APS, Guimarães MDC. Fatores associados ao abandono do tratamento psiquiátrico em um centro de referência em saúde mental em Belo Horizonte. Rev Bras Psiquiatr. 2005;27(2):113-8.

42. Ribeiro MS, Alves MJM, Vieira EMM, Silva PM, Lamas CVD-B. Fatores associados ao abandono de tratamento em saúde mental em uma unidade de nível secundário do Sistema Municipal de Saúde. J Bras Psiquiatr. 2008;57(1):16-22.

43. Perkins R. What Constitutes Success? [Editorial]. Br J Psychiatry. 2001;179:9-10

44. Grunebaum M, Luber P, Callaham M, Leon AC, Olfson M, Portera L. Predictors of missed appointments for psychiatric consultations in a primary care clinic. Psychiatr Serv. 1996;47(8):848-52.

45. Killaspy H, Banerjee S, King M, Lloyd M. Prospective controlled study of psychiatric out-patient non-attendance. Characteristics and outcome. $\mathrm{Br} \mathrm{J}$ Psychiatry. 2000;176:160-5.

46. Siqueira MM, Garcia MLT, Souza RS. O impacto das faltas às consultas em um programa de dependentes de álcool. J Bras Psiquiatr. 2005;54(2):114-9.

47. Percudani M, Belloni G, Contini A, Barbui C. Monitoring community psychiatric services in Italy: differences between patients who leave care and those who stay in treatment. Br J Psychiatry. 2002;180:254-9.

48. Bao Y, Duan, N, Fox SA. Is some provider advice on smoking cessation better than no advice? An instrumental variable analysis of the 2001 National Health Interview Survey Health Serv Res. 2006;41(6):2114-35.

49. Haro JM, Suarez D, Novick D, Brown J, Usall J, Naber D, et al. Three-year antipsychotic effectiveness in the outpatient care of schizophrenia: observational versus randomized studies results. Eur Neuropsychopharmacol. 2006;17(4):235-44 